issues du côté portugais . . - - semblent dire « la vérité ». Ainsi, la population vivant dans les zones libérées du Frelimo est évaluée à 1800000 personnes «organisées en coopératives », parce que ce chiffre (p. 18 et note 815 p. 359) est dans un article de la presse... danoise, envoyé par l'Ambassade portugaise dans ce pays au ministère des Affaires étrangères et se retrouve donc aujourd'hui dans un dossier de l'Arquivo Histórico-Diplomático (en réalité, probablement 300000 personnes vivaient totalement en «zones libérées »). Ainsi, l'émeute de Mueda n'aurait rien à voir avec les Macondes du Tanganyika (p. 133 et note 523 p. 344) parce que le consul belge à Lourenço Marques, en un rapport fort mal renseigné capté par la PIDE, le dit, et dit aussi qu'il s'agissait en fait d'une révolte contre la culture forcée du coton. Or, il n'y avait aucune culture forcée du coton sur le planalto maconde et l'émeute eut lieu à l'occasion d'une réunion publique en présence d'un délégué maconde du Tanganyika lié aux associations de l'émigration mozambicaine dans ce pays. On trouvera des dizaines d'exemples similaires dans le livre. L'auteur ne discute pas ses sources, il les accumule simplement. De même, des erreurs de noms sont fidèlement reproduites, parce qu'elles figurent dans les archives (ainsi «John Marvem» au lieu de John Marcum, p. 350).

Cette très grande carence méthodologique dans l'ouvrage est pourtant à mettre en rapport avec un phénomène plutôt positif et que souligne Adriano Moreira dans sa préface: «Le nombre d'officiers des Forces armées qui cherchent la consécration de titres universitaires, s'accroît» (p. 15). Effectivement, l'auteur est militaire, diplômé en «sciences militaires » de l'Académie militaire en même temps qu'il est docteur en histoire contemporaine d'une université privée, l'Universidade Portucalense. On ne peut que se réjouir de cette «tendance universitaire » chez certains militaires, qu'ils aient connu ou non la guerre coloniale. Mais on peut aussi questionner leur encadrement et leur préparation pour devenir docteur en sciences sociales. Pourtant le Portugal, moderne et démocratique, a aujourd'hui d'excellents centres d'études africaines. Plutôt que de créer l'« université des Forces armées » souhaitée par Adriano Moreira, mieux vaudrait utiliser les outils excellents existant dans les universités publiques.

Mai 2005, Michel Gahen

\title{
Álvaro Nóbrega, A luta pelo poder na Guiné-Bissau, Lisbonne, Univer- sidade técnica de Lisboa, Instituto superior de ciências sociais e políti- cas, 2003, 334 p., bibl., index, ISBN : 972-8726-19-8.
}

Pourquoi publier une thèse de mestrado, que l'on peut comparer en France, selon les cas, aux anciennes thèses de troisième cycle ou aux mémoires de masters de cinquième année d'université ? La recherche ici présentée aurait en effet gagné à être approfondie en thèse de doctorat, si possible en élargissant le cadre institutionnel où l'auteur a "vécu ", à savoir l'ISCSP, à d'autres milieux académiques et scientifiques. La recherche menée a été importante, malgré des conditions difficiles. Les sources, cependant, restent lacunaires, des travaux considérables de langue anglaise ne semblant pas connues (livres de P. Chabal, R. Chilcote, J. Forrest, R. Lobban, L. Rüdebeck). Il en va de même pour ceux, moins nombreux, de 
langue française (comme les articles de Lusotopie, en accès intégral, libre et gratuit sur le site internet de la revue ; ceux de Politique Africaine; les ouvrages de Rüdebeck en français). En langue portugaise enfin, on s'étonne de ne rien trouver de P. Havik ou D.L. Handem dans la bibliographie.

Mais le problème n'est pas là. Une grande partie du travail consiste en une description très classique des «ethnies » et de leurs religions ( $1^{\mathrm{e}}$ partie), sans que, malgré un effort de cartographie important, l'hétérogénéité sociale dans le rapport à l'État moderne ne soit clairement étudié. L'auteur a raison de replacer la violence dans son cadre historique ( $2^{\mathrm{e}}$ partie), mais d'une part on aurait aimé que cette «violence » fût mise en rapport avec la formation sociale des peuples présentés (sociétés hiérarchisées et centralisées, ou segmentaires voire acéphales) et d'autre part, on est gêné de l'approche en terme de "longue tradition de violence », comme si la violence était un atavisme culturaliste et non point un produit social contextualisé.

Outre l'hétérogénéité du pays - constat statistique - on voit mal l'historicité de la crise du rapport à l'État, et notamment la contradiction «fondatrice » entre un «lieu de l'État»(Bissau) situé loin des sociétés les plus aptes à entrer en contact fécond avec lui (les sociétés musulmanes de l'Est). En effet, le Portugal catholique avait largement utilisé les auxiliaires musulmans pour vaincre les sociétés côtières acéphales. La guerre de libération n'est ainsi pas assez vue comme guerre civile non créatrice, en elle-même, d'un sentiment national.

Une sociologie historique et politique des élites était également nécessaire. Finalement, ce qui restera de plus utile dans ces 334 pages, est la troisième et dernière partie, surtout les pages qui traitent de la situation postérieure au 14 novembre 1980, en tant que chronique politique de l'instabilité endémique du pays. Une étude plus poussée du Partido de Renovação social (PRS) aurait été fort utile : est-ce l'apparition d'une expression politique plébéienne, au-delà des aspects ethniques? Par ailleurs, le formidable retournement de situation après les élections de 1999, qui voit le «PAIGC résiduel» sauvegarder de l'influence surtout en zones musulmanes - traditionnellement réticentes pendant la guerre de libération -, n'est pas entrevu, et repose pourtant la question de l'« alliance structurelle » entre les sociétés fortement hiérarchisées et l'État moderne. La Guinée-Bissau, si petite et si complexe, peut être analysée, dans sa crise permanente, comme un modèle invivable de néopatrimonialisme pauvre. L'auteur aurait gagné à mieux connaître la littérature française sur le thème: J.-F. Bayart, J.-F. Médard, J.-P. Olivier de Sardan, etc.

Enfin, certaines pages sont étonnantes dans une thèse, comme ces pages 44-47 où l'auteur dérive vers des recommandations en vue d'augmenter l'influence du Portugal en Guinée-Bissau, allant jusqu'à proposer des manœuvres militaires conjointes Portugal/Guinée (que naturellement la Guinée-Bissau post-cabralienne n'acceptera jamais!). Que l'on sache, l'ISCSP n'est plus l'École coloniale et une thèse n'est pas un rapport de consultance pour le ministère ...Et je n'écris point cela parce que je suis français! D'ailleurs, il y aurait eu une analyse extrêmement intéressante à produire sur la farouche rivalité politique Portugal/CPLP versus France/UMEOA dans les pourparlers de paix de 1998-99, qui fit capoter un premier accord, mais se solda in fine par une claire «victoire » du Portugal et par la montée d'un sentiment anti-français à Bissau, vu le soutien accordé par la France 
au dictateur Nino Vieira et à l'intervention sénégalaise ressentie par tous comme « péri-française »!

L'ISCSP et l'auteur avaient sans doute des raisons institutionnelles de publier ce mestrado le plus vite possible. Mais on regrettera, sur le plan scientifique, cette précipitation, car on reste sur sa faim relativement au besoin maintenu d'une analyse, sur la durée, du rapport au pouvoir en Guinée-Bissau. Un pas a été fait en ce sens par Fafali Koudawo, Cabo Verde e Guiné-Bissau, da democracia revolucionária à democracia liberal, Bissau, INEP, 2001, 230 p. ("Kacu Martel », 14) et par Lars RÜDEBEGK, en deux versions légèrement différentes, anglaise et portugaise : On Democracy's Sustainability. Transition in Guinea-Bissau, Stockholm, SIDA, 2001, 125 p. ("Sida Studies », 4) et Colapso e reconstrução política na Guinée-Bissau, 1998-2000. Um estudo de democratização difíil, Uppsala, Nordiska Afrikainstitutet, 2001, 124 p., ISBN : 91-7106-482-6, livres qui auraient impérativement dû entrer dans la bibliographie de l'auteur.

Souhaitons qu'Álvaro Nóbrega remette main à l'ouvrage, joignant plus profondément histoire, anthropologie politique et sciences politiques.

Mai 2005, Michel Gahen

\section{Luís Quintais, As guerras coloniais portuguesas e a invenção da História, Lisboune, Imprensa Giências Sociais, novembre 2000, 163 p., ISBN: 972- 671-065-0 ("Estudose Instigações »)}

Devais-je rédiger cette note de lecture portant sur un domaine qui ne m'est guère familier? En effet, ce livre, publication en novembre 2000 d'une thèse de troisième cycle (mestrado), soutenu en février 1998, se situe avant tout dans le champ de l'anthropologie médicale - même si l'anthropologie des émotions et une étude de la mémoire y ont leur place. Je ne suis donc pas en mesure de commenter la bibliographie et avoue avoir été dépassée par les trois premiers chapitres qui retracent l'histoire des courants de la psychiatrie moderne et reviennent sur l'évolution des définitions du concept de «désordre de stress post-traumatique ». Ma présentation en est, dès le départ, quelque peu biaisée.

Le thème était alléchant: l'auteur revenait sur deux visions différentes, voire antagoniques, de l'histoire: l'une qui réfute toute possibilité de réécriture de l'histoire et l'autre qui pose que toute évocation du passé est systématiquement une réécriture. S'appuyant sur cette dernière, il constate qu'il serait dès lors envisageable de trouver une solution thérapeutique aux maux dont souffrent les vétérans des guerres coloniales, solution permettant une réappropriation du temps et qui se révèlerait efficace y compris sur le plan politique et moral. En utilisant le vocabulaire médical, on inventerait, modèlerait une évocation de cette période historique. Suivant cet objectif, l'auteur participa durant six mois aux séances thérapeutiques du foyer militaire de la Croix Rouge et de l'Association des handicapés des Forces armées (ADFA).

L'auteur se réfère à Afonso de Albuquerque, qu'il cite abondamment et dont le parcours ne peut, selon lui, être dissocié de l'étude des PTSD (post-traumatic stress disorder) au Portugal. Ce psychiatre établit les premiers diagnostics de PTSD en 